Servicio de Neurología, Hospital Dr. Sótero del Río. Santiago,

Chile.

aAlumno de Medicina, Facultad de Medicina, Pontificia Universidad Católica de Chile. Santiago, Chile.

Fuente de apoyo financiero: ninguna.

Recibido el 12 de septiembre de 2013, aceptado el 4 de marzo de 2014 .

Correspondencia a: Dra. Eva Retamal Riquelme. Servicio de Neurología, Hospital Dr. Sótero del Río. Concha y Toro 3459, $3^{\circ}$ piso. Santiago, Chile. eiretama@uc.cl

\section{Neurotoxicidad secundaria a metronidazol: un efecto adverso reversible. Caso clínico}

\author{
EVA RETAMAL-RIQUELME, HERNÁN SOTO-SAN MARTÍNa, \\ JOSÉ VALLEJOS-CASTRO, DANIEL GALDAMES-POBLETE
}

\section{Reversible neurotoxicity secondary to metronidazole report of one case}

\begin{abstract}
Metronidazole can cause adverse effects both in the central and peripheral nervous system. We report a 34-year-old female who presented a reversible cerebellar syndrome and peripheral neuropathy as an adverse effect associated with the use of metronidazole. Brain magnetic resonance imaging (MRI) showed hyperintense T2 and FLAIR bilateral symmetrical cerebellar lesions, without contrast enhancement or mass effect, isointense in diffusion-weighted imaging and hypointense in apparent diffusion coefficient sequences. Also, electrophysiological evaluation was consistent with axonal polyneuropathy. She had received metronidazole for a liver abscess during 49 days. After discontinuation of metronidazole, she had rapid regression of cerebellar symptoms and normalization of MRI, with subsequent disappearance of peripheral symptoms. The brain MRI, electromyography and nerve conduction studies performed at 35 months later showed complete resolution of the lesions. Although metronidazole neurotoxicity is a rare event, it must be borne in mind because the prognosis is usually favorable after stopping the drug.
\end{abstract}

(Rev Med Chile 2014; 142: 386-390)

Key words: Cerebellar diseases; Metronidazole; Peripheral Nervous System diseases.
E 1 metronidazol es un antibiótico ampliamente usado en la práctica clínica, especialmente en infecciones por anaerobios y protozoos. Existen múltiples reportes de casos de neurotoxicidad inducida por metronidazol con manifestaciones tanto centrales como periféricas: encefalopatía, crisis epilépticas, síndrome cerebeloso, neuropatía sensitiva, neuropatía autonómica y neuropatía óptica, entre otros ${ }^{1,2}$. Estos efectos adversos se consideran infrecuentes, aunque no se cuenta con datos precisos al respecto ${ }^{3}$. Se han propuesto como posibles factores de riesgo presencia de disfunción hepática ${ }^{4}$, el tiempo de uso del fármaco y la dosis total acumulada ${ }^{5}$, aunque una revisión sistemática reciente, limitada a casos con compromiso solamente de sistema nervioso central (SNC), no mostró asociación con dosis ni duración del tratamiento ${ }^{3}$.

Debido a lo mencionado, al uso frecuente de metronidazol y a la ausencia de publicaciones de habla española de este tema, nos ha llevado a comunicar la paciente que a continuación se detalla.

\section{Caso clínico}

Mujer de 34 años, dueña de casa, con antecedentes de siete semanas de evolución de lesiones quísticas, hepáticas, múltiples abcesos, con drenaje quirúrgico y tratamiento prolongado sin antibióticos. Fue dada de alta con terapia antibiótica. Consultó en el Servicio de Urgencia del Hospital 
Sótero del Río por un cuadro de inicio progresivo, de cinco días de evolución, caracterizado por náuseas, vómitos, trastorno del habla e inestabilidad de la marcha invalidante. Al ingreso destacaban signos vitales normales, ausencia de cefalea, fiebre o signos meníngeos; en exámenes de laboratorio, GOT y GPT normales $(21$ y $13 \mathrm{U} / \mathrm{L}$, respectivamente), aumento de GGT (204 UI/L, rango normal 5-36) y fosfatasa alcalina (376 U/L, rango normal 35-104); hipoalbuminemia (2,6 g/dL), bilirrubina total normal $(0.37 \mathrm{mg} / \mathrm{dL})$ y tiempo de protrombina prolongado $(60 \%$, INR 1,51$)$. Al examen neurológico, la paciente estaba orientada, atenta, con disartria escandida, sin nistagmus ni alteración de nervios craneales. Al examen motor no había paresia, los reflejos osteotendíneos eran vivos, con tono normal y reflejos plantares flexores. No se pesquisaron alteraciones sensitivas. En las pruebas cerebelosas presentaba dismetría leve en extremidades superiores y marcada ataxia axial y de la marcha que le impedían sentarse o caminar sin asistencia.
La evaluación inicial con tomografía computadorizada de encéfalo y estudio de líquido cefalorraquídeo no mostraron alteraciones (citoquímico: aspecto claro, incoloro; glucosa 52 $\mathrm{mg} / \mathrm{dL}$-con glicemia $81 \mathrm{mg} / \mathrm{dL}-$; proteínas 0,24 g/L; sin leucocitos; Gram sin bacterias ni células, cultivo negativo). La resonancia magnética (RM) de encéfalo mostró lesiones bilaterales, simétricas, hiperintensas en secuencias T2 y FLAIR localizadas en el núcleo dentado y la región dorsal del puente, sin efecto de masa, sin captación de contraste, isointensas en difusión e hiperintensas en ADC (Figura 1).

En base a los estudios mencionados, se descartó la posibilidad de causa infecciosa, tumoral e inflamatoria, planteándose como causa más probable la toxicidad farmacológica. Se revisó el uso de los medicamentos desde el diagnóstico del absceso hepático, destacando el uso de metronidazol durante 49 días. La dosis total acumulada de metronidazol fue 73,5 g. Además, recibió otros antibióticos asociados: al inicio, ceftriaxona ( $2 \mathrm{~g}$

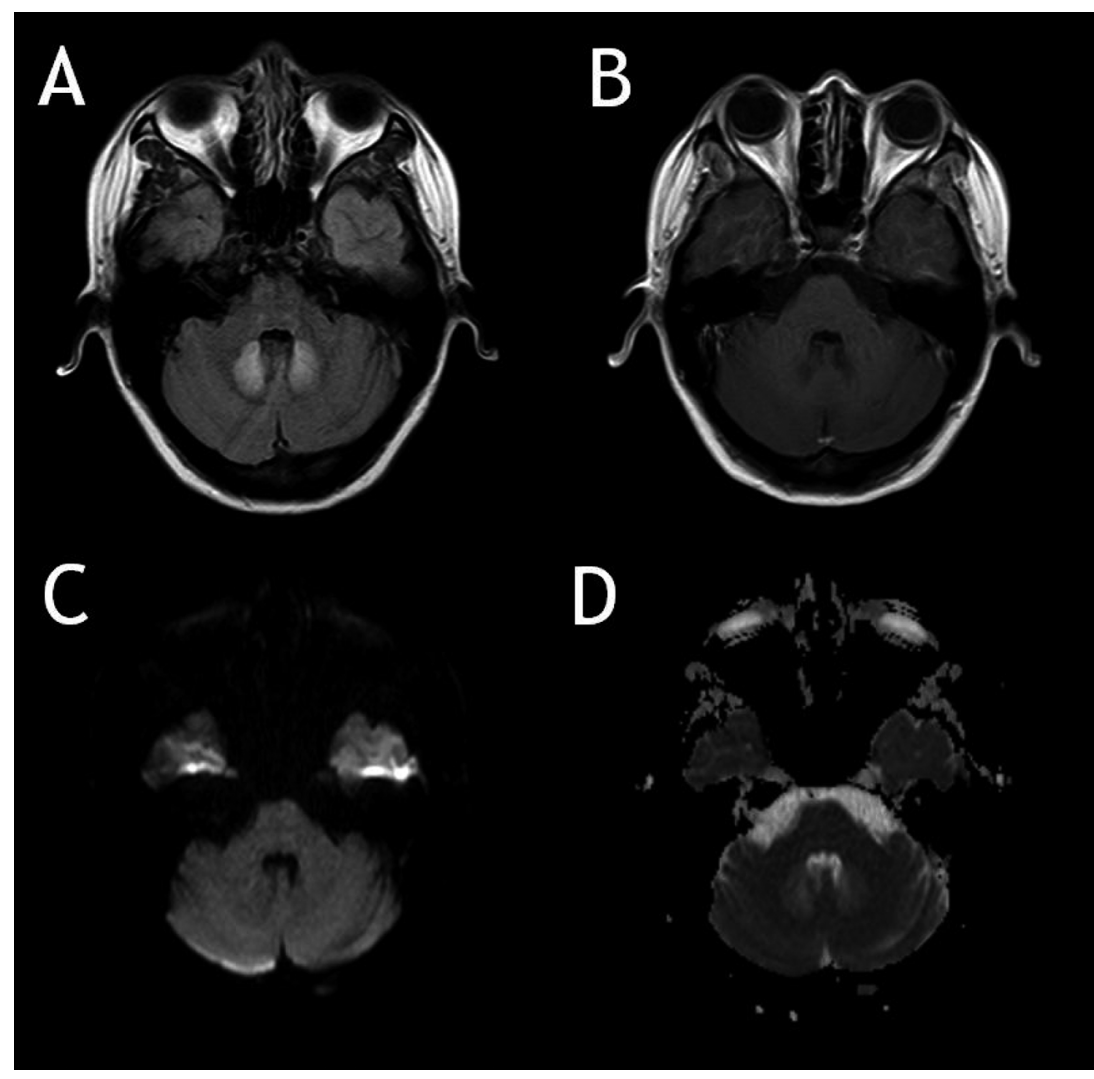

Figura 1. Resonancia magnética de encéfalo realizada al momento del diagnóstico. Lesiones hiperintensas bilaterales y simétricas en núcleos dentados de cerebelo y región dorsal de puente en secuencia FLAIR (A), sin efecto de masa, sin captación de contraste en $\mathrm{T} 1$ con gadolinio (B), isointensas en difusión (C) e hiperintensas en ADC (D). 
al día por 29 días), ampicilina ( $1 \mathrm{~g}$ cada $6 \mathrm{~h}$ por 3 días), luego linezolid (600 mg cada $12 \mathrm{~h}$ por 15 días), y finalmente ciprofloxacino (750 mg cada 12 $\mathrm{h}$, por 20 días) hasta su ingreso. Ante la sospecha de neurotoxicidad por metronidazol, se suspendió el fármaco, cambiándose el tratamiento antibiótico a ertapenem y linezolid.

La paciente evolucionó con rápida regresión del cuadro cerebeloso, que se resolvió completamente al quinto día de suspender el metronidazol. Ulteriormente la paciente refirió disestesias y alodinia palmoplantares, sin déficit objetivable al examen neurológico. Dado que impresionaba sugerente de compromiso neuropático, se inició tratamiento con amitriptilina, con mejoría parcial de los síntomas. No se efectuó estudio neurofisiológico.

En un control a los 2 meses después de la suspensión de metronidazol, la paciente no presentaba alteraciones cerebelosas aunque persistía con parestesias en palmas y plantas. Al examen neurológico destacaba la presencia de reflejos osteotendíneos vivos, sin signos de disfunción cerebelosa ni déficit de vías largas. La RM de control realizada en ese momento mostró resolución completa de las lesiones (Figura 2).

Evaluada 8 meses después, la paciente sólo refería parestesias distales en extremidades inferiores, con examen neurológico dentro de límites normales. Se efectuó estudio electrofisiológico (electromiografía y velocidades de conducción nerviosa, EMG/VCN) de extremidades inferiores, evidenciándose una polineuropatía (PNP) axonal sensitiva leve, debido a la ausencia de potenciales sensitivos en ambos nervios surales, los cuales reaparecieron en el control de EMG/ VCN realizado 3 meses después, estando dentro de rango normal, al igual que los demás parámetros del estudio.

En su último control, 35 meses tras suspender el fármaco, la paciente era capaz de realizar sus actividades habituales, refería mínima inestabilidad que le impedía andar en bicicleta y persistencia de disestesias plantares en el tercio distal, sin déficit

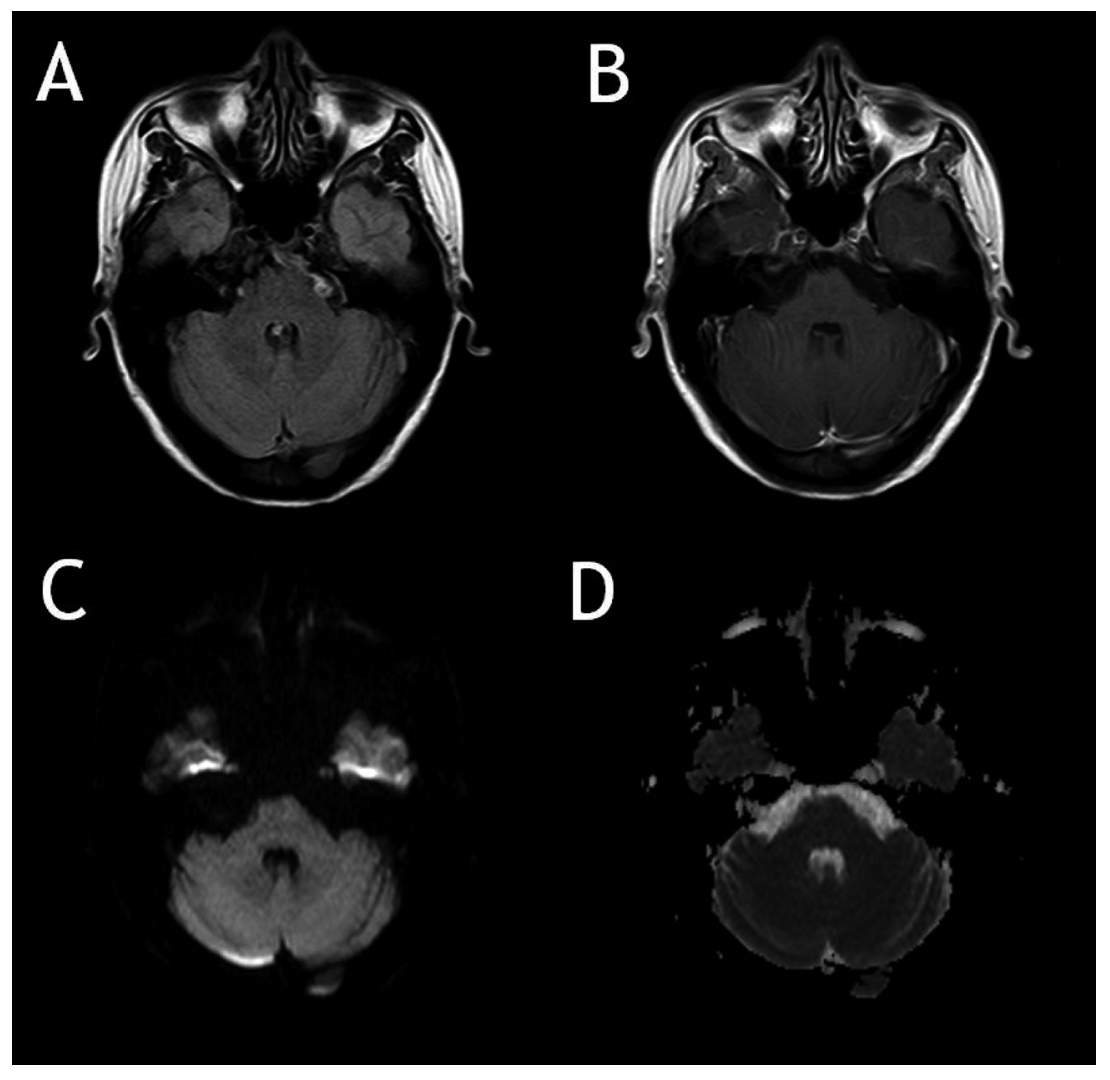

Figura 2. Resonancia magnética de encéfalo de control, 8 semanas después de suspensión de metronidazol. Se observa resolución completa de lesiones. Secuencias: A) FLAIR; B) T1 con gadolinio; C) Difusión; D) ADC. 
objetivable al examen físico. Se realizó una nueva RM de encéfalo y EMG/VCN, ambas sin hallazgos patológicos.

\section{Discusión}

La neurotoxicidad por metronidazol tiene múltiples manifestaciones clínicas entre las cuales destacan el compromiso cerebeloso y la PNP sensitiva. Al suspender el fármaco, en la gran mayoría de los casos, los síntomas son reversibles, especialmente los cerebelosos ${ }^{1}$.

Nuestra paciente presentó inicialmente un síndrome cerebeloso vermiano y PNP en contexto de uso prolongado de metronidazol. Puesto que el metronidazol se metaboliza a nivel hepático ${ }^{6}$, es posible que su patología de base fuera un factor predisponente.

El síndrome cerebeloso secundario a metronidazol se asocia a hallazgos típicos en RM, que muestra lesiones hiperintensas en secuencias T2, bilaterales, simétricas, localizadas en el núcleo dentado de cerebelo (localización más frecuente), mesencéfalo, dorso de puente, bulbo, cuerpo calloso y sustancia blanca cerebral. En difusión, las lesiones aparecen iso o hiperintensas, y rara vez son hipointensas en la secuencia ADC (apparent diffusion coefficient). En los casos en que se dispone de imágenes de control, éstas son reversibles, salvo cuando inicialmente la lesión fue hipointensa en $\mathrm{ADC}^{7,3}$. La fisiopatología exacta de este cuadro se desconoce. Dado su carácter reversible, se ha planteado que las lesiones cerebelosas pudieran corresponder, entre otras cosas, a edema vasogénico ${ }^{5,8}$.

El estudio con RM apoya al diagnóstico, y orienta el pronóstico. En una revisión sistemática de toxicidad de SNC secundaria a metronidazol, todos los casos con signología cerebelosa presentaron alteraciones en neuroimágenes y $83 \%$ evolucionaron con resolución completa de éstas en las RM de control. Desde el punto de vista clínico, la mayoría presentó resolución parcial (29\%) o completa $(65 \%)$ de su sintomatología tras suspender el fármaco ${ }^{3}$, lo que resalta la importancia de considerar la toxicidad inducida por fármacos en el diagnóstico diferencial de síntomas neurológicos en pacientes tratados con metronidazol. Los hallazgos en secuencias de difusión y ADC se correlacionaron con el grado de reversibilidad de las lesiones ${ }^{7}$.
Entre los diagnósticos diferenciales para pacientes con encefalopatía aguda según los hallazgos en $\mathrm{RM}$, la presencia de lesiones generalizadas, simétricas, que pueden comprometer corteza cerebral y núcleos basales suele corresponder a patologías tóxicas y metabólicas adquiridas, tales como encefalopatía hepática, hipoglicémica, intoxicación por monóxido de carbono, desmielinización osmótica, toxicidad por ciclosporina, entre otras 9 .

Respecto a las lesiones más frecuentes en encefalopatía secundaria a metronidazol, éstas son multifocales, simétricas, comprometen sustancia blanca y gris y carecen de efecto de masa significativo, por lo que es necesario considerar entre los diagnósticos diferenciales: enfermedades inflamatorias desmielinizantes de SNC (encefalomielitis aguda diseminada, esclerosis múltiple), compromiso secundario enfermedades autoinmunes sistémicas (por ejemplo, síndrome de Sjögren), patologías infecciosas (encefalitis virales, meningoencefalitis por Listeria), paraneoplásicas (cerebelitis), metabólicas (encefalopatía de Wernicke, desmielinización osmótica), y otras intoxicaciones (monóxido de carbono, bromuro de metilo) ${ }^{5,8}$.

En los casos publicados en que coinciden síntomas cerebelosos y PNP en un mismo paciente, hay un perfil de instalación y evolutivo similar (inicio con el cuadro cerebeloso, luego se agrega el compromiso polineuropático, evolucionando con rápida resolución del cuadro cerebeloso, y persistencia de los síntomas neuropáticos por semanas e incluso meses ${ }^{2,5,8,10,11}$, lo que concuerda con la evolución de nuestra enferma.

Para establecer una relación de causalidad en neuropatías tóxicas, debe haber asociación temporal entre inicio de los síntomas y exposición al tóxico. No obstante, la progresión de la neuropatía puede ser durante semanas o meses, incluso después de eliminar el tóxico (efecto de inercia), lo que sucedió en nuestro caso ${ }^{12}$.

En conclusión, el amplio uso de metronidazol en la práctica diaria obliga a tener presente las complicaciones neurológicas de este antibiótico. Ante la aparición de alteraciones neurológicas centrales es necesario realizar los estudios de neuroimagen correspondientes (RM), cuyos hallazgos orientan a este diagnóstico. El estudio de síntomas polineuropáticos debe complementarse con la realización de EMG/VCN. La suspensión inmediata está indicada para lograr una mejoría de 
los síntomas neurológicos, centrales y periféricos.

Si bien la neurotoxicidad por metronidazol es un evento poco frecuente, es muy importante considerarlo pues en la gran mayoría de los casos el pronóstico es muy favorable tras suspender el fármaco. Este caso, el con mayor seguimiento clínico disponible en la literatura, ilustra el carácter reversible de esta patología, si se efectúa la suspensión oportuna del metronidazol.

Agradecimientos: Al Dr. Marcelo Rodríguez Pérez, por su ayuda en la redacción del manuscrito final.

\section{Referencias}

1. Sarna JR, Brownell AK, Furtado S. Cases: Reversible cerebellar syndrome caused by metronidazole. CMAJ 2009; 181 (9): 611-3.

2. Park KI, Chung JM, Kim JY. Metronidazole neurotoxicity: sequential neuroaxis involvement. Neurol India 2011 (1); 59: 104-7.

3. Kuriyama A, Jackson JL, Doi A, Kamiya T. Metronidazole-induced central nervous system toxicity: a systematic review. Clin Neuropharmacol 2011; 34 (6): 241-7.

4. Horlen CK, Seifert CF, Malouf CS. Toxic metronidazoleinduced MRI changes. Ann Pharmacother 2000; 34 (11): 1273-5.
5. Patel K, Green-Hopkins I, Lu S, Tunkel AR. Cerebellar ataxia following prolonged use of metronidazole: case report and literature review. Int J Infect Dis 2008; 12 (6): 111-4.

6. Lamp KC, Freeman CD, Klutman NE, Lacy MK. Pharmacokinetics and pharmacodynamics of the nitroimidazole antimicrobials. Clin Pharmacokinet 1999; 36 (5): 353-73.

7. Kim E, Na DG, Kim EY, Kim JH, Son KR, Chang KH. MR imaging of metronidazole-induced encephalopathy: lesion distribution and diffusion-weighted imaging findings. AJNR Am J Neuroradiol 2007; 28 (9): 1652-8.

8. Ahmed A, Loes DJ, Bressler EL. Reversible magnetic resonance imaging findings in metronidazole-induced encephalopathy. Neurology 1995; 45 (3 Pt 1): 588-9.

9. Sharma P, Eesa M, Scott JN. Toxic and acquired metabolic encephalopathies: MRI appearance. Am J Roentgenol 2009; 193 (3): 879-86.

10. Heaney CJ, Campeau NG, Lindell EP. MR imaging and diffusion-weighted imaging changes in metronidazole (Flagyl)-induced cerebellar toxicity. AJNR Am J Neuroradiol 2003; 24 (8): 1615-7.

11. Toumi S, Hammouda M, Essid A, Medimagh L, Slamia LB, Laouani-Kechrid C. Metronidazole-induced reversible cerebellar lesions and peripheral neuropathy. Med Mal Infect 2009; 39 (12): 906-8.

12. Misra UK, Kalita J. Toxic neuropathies. Neurol India 2009; 57 (6): 697-705. 